\title{
Maximum Weight Basis Decoding of Convolutional Codes
}

\author{
Suman Das * Elza Erkip Joseph R. Cavallaro Behnaam Aazhang \\ suman@rice.edu elza@rice.eduｃavallar@rice.eduａaz@rice.edu \\ Department of Electrical and Computer Engineering \\ Rice University, MS 366 \\ 6100 South Main Street \\ Houston, TX 77005, USA \\ Phone: (713)-527-4719 Fax:(713)-524-5237
}

\begin{abstract}
The effectiveness of the convolutional code increases with the constraint length of the code. Unfortunately the decoding complexity of Viterbi algorithm grows exponentially with the constraint length. In this paper we propose a suboptimal decoding method based on the calculation of the maximum weight basis of the convolutional code. We extend the algorithm to incorporate the ideas of list decoding method. The complexity of the algorithm grows only quadratically with the constraint length and the performance of the algorithm is comparable to the optimal Viterbi decoding method.
\end{abstract}

\section{Introduction}

The use of convolutional codes is a well-known error-control technique for reliable communication of digital data over a noisy channel. Convolutional codes have become a popular option once Viterbi introduced his optimal yet computationally efficient decoding algorithm [1]. Viterbi algorithm is the maximum likelihood decoding algorithm. Specifically, this algorithm tries to minimize the probability of decoding a sequence of received signal to an incorrect codeword or information bits. Unlike most of the block codes and their decoding algorithms, the complexity of the Viterbi algorithm does not grow exponentially with the block length of the code but only linearly. This makes it an attractive option for physical implementation.

The convolutional codes differ from the block codes in that each encoding operation depends on current as well a number of past information bits. This number is the constraint length of the code. The strength of the convolutional codes is not only given by the rate of the code, but also the length of the convolutional codes. It is desirable to have a large constrained length convolutional code to maximize the capability to recover from error. However the complexity of Viterbi algorithm grows exponentially with the constraint length. This precludes large constraint length convolutional codes from being practically implemented, especially in a system with real time demands like a wireless receiver.

There are several other decoding techniques $[2,3]$ for convolutional codes that have been proposed in the literature. Many of them are sub-optimum decoding techniques that sacrifice performance to cut down the computational complexity. The dynamic programming algorithms [4] are designed so that their computation cost is independent of the constraint length. However the number of computation that needs to be done is unpredictable. This is not usually acceptable for realtime implementation. Moreover at low signal to noise ratio (SNR) the computations are not only heavy but can lead to decoding failure.

In this paper we propose a suboptimal decoding algorithm for convolutional codes based on the maximum weight basis of the code. The initial decoding idea is similar to generalized Dijkstra's algorithm [5] used for block codes by several researchers [6,7]. However the search problem is much simpler in case of a linear

\footnotetext{
*Research supported by Nokia Inc., by the Texas Advanced Technology Program under grants 1995-\#003604-049 and 1997\#003604-044, and by NSF under grants NCR-9506681 and CDA-9617383.
} 
block code than a convolutional code. Moreover we incorporate the idea of list decoding [8] to this algorithm to obtain better results.

The rest of the paper is organized as follows. In section two we provide our system details. We also briefly describe the Viterbi algorithm and its drawback, and motivate our algorithm with a numerical example. We then introduce the idea of maximal weight basis and describe an algorithm to calculate it. We also describe a computationally efficient technique to compute the M-largest weight basis and from there the final decoded data. We end with some concluding remarks and ideas for future work.

\section{System description}

We will assume that the raw information bits, $\mathbf{b}$, are encoded by a convolutional encoder. Usually for transmission over a channel, the preferred alphabet are the antipodal signals $( \pm 1)$. Thus the convolutionally encoded $\{0,1\}$ bit sequences are mapped to antipodal symbols. The receiver receives the original symbols corrupted by noise $\mathbf{r}=\sqrt{\mathcal{E}} \mathbf{d}+\eta$, where $\mathcal{E}$ is the received energy of the signal, $\mathbf{d}$ is the coded bit sequence and $\eta$ is the noise. In our system we will assume that the noise is additive white gaussian noise with mean zero and variance $\sigma^{2}$. The receiver will have to estimate the original information bits from the received symbols.

\subsection{Viterbi decoding}

If we consider an additive gaussian channel then the decoding rule that minimizes the probability of error is given by

$$
\hat{\mathbf{b}}=\arg \max _{\mathbf{b}} p(\mathbf{b} \mid \mathbf{r})
$$

Since there is a one-one correspondence between the information bits and the coded words, it can be shown that the above optimization criteria is equivalent to finding a codeword sequence that has minimum Euclidean distance from the received signal. The optimum decoding technique is the maximum likelihood sequence estimator. Essentially we should explore the code trellis to generate all the possible codeword sequences and enumerate the associated probability of error. If the block length of the code is $N$, and the code rate is $R$, there are $2^{N R}$ possible codewords which should be considered. Viterbi algorithm describes an iterative method to calculate the likelihood over the various stages of the trellis. This reduces the complexity of the algorithm to linear in the codeword length. However at every stage Viterbi algorithm has to calculate the likelihood of each state. The number of states is exponential in the size of the constraint length of the code. Thus Viterbi algorithm for a convolutional code of constraint length $\kappa$ and codeword length of $N$ requires $N 2^{2(\kappa+1)}$ operations indicating that the complexity becomes prohibitively large for large constraint lengths. We will describe a suboptimal channel decoding algorithm which has quadratic complexity in the constraint length of the convolutional code.

\subsection{Motivation of the maximum weight basis}

In order to motivate the problem, let us consider a numerical example of a simple rate $2 / 3$ even parity check code. In this code for every two bits of information three coded bits are produced where the first two bits are the original information bits and the third bit is the modulo- 2 sum of the two information bits. Let the received signal be the coded bits corrupted by additive gaussian noise. The information bits are $\mathbf{b}=\{1,0\}$ and thus the transmitted codeword is $\mathbf{d}=\{1,-1,1\}$. Let the received signal be $\mathbf{r}=\{7.5,0.5,7.5\}$. We use maximum likelihood sequence estimation (MLSE) method to estimate the information bits. In this particular example the decoder would correctly recognize the information bits as $\{1,0\}$.

If the transmitted bits were unconstrained binary sequence, then the MLSE estimate is simply the sign estimation of the received sequence $\hat{\mathbf{d}}=\operatorname{sgn}(\mathbf{r})$ and the decoding has trivial computational complexity. However in this method of decoding it is not always guaranteed that the estimated bit sequence will be a codeword. The decoding complexity becomes non-trivial when we have to restrict our search to codewords only. To summarize, reduction in decoding complexity can be achieved if we can make our search unconstrained. 
In the maximum likelihood sequence estimation not only do we estimate the codeword that is most likely but also the likelihood value corresponding to that codeword. We define the likelihood ratio corresponding to the $j^{\text {th }}$ bit of the codeword $\hat{d}_{j}$ as

$$
\phi_{j}=\frac{\operatorname{Pr}\left(r_{j} \mid \hat{d}_{j}=1\right)}{\operatorname{Pr}\left(r_{j} \mid \hat{d}_{j}=-1\right)},
$$

and the likelihood ratio corresponding to the estimated codeword $\hat{\mathbf{d}}$ as

$$
\phi(\hat{\mathbf{d}})=\prod_{j=1}^{N} \phi_{j} .
$$

The MLSE algorithm identifies the codeword for which this likelihood ratio is maximum. The value of the likelihood ratio measures our confidence in the estimated codeword based on the received signal. A large likelihood ratio, corresponding to a codeword, implies that the codeword is very likely to be the optimal codeword. We can write our decoding optimization problem as

$$
\hat{\mathbf{d}}=\arg \max _{\mathbf{d} \in \mathcal{C}} \phi(\mathbf{d}),
$$

where $\mathcal{C}$ represents the set of codewords. We note that the information bits can take any arbitrary binary values while the parity bits are uniquely determined by the information bits. If $I$ represent the locations corresponding to the information bits and $D$ represent the locations corresponding to the parity bits, then we can partition the codeword as $\mathbf{d}_{I}$, representing the information bits, and $\mathbf{d}_{D}$ the parity bits. We can rewrite (1) as

$$
\begin{aligned}
\hat{\mathbf{d}} & =\arg \max _{\left\{\mathbf{d}_{I}, \mathbf{d}_{D}\right\} \in \mathcal{C}} \prod_{j=1}^{N} \phi_{j} \\
& =\arg \max _{\left\{\mathbf{d}_{I}, \mathbf{d}_{D}\right\} \in \mathcal{C}} \prod_{i \in I} \phi_{i} \prod_{j \in D} \phi_{j}
\end{aligned}
$$

The first part of the optimization involving the information bits is unconstrained, while the second part of the optimization is constrained but cannot be ignored for the correct results. In our numerical example if we consider only the first part of the optimization to save computation then the information bit sequence with largest likelihood ratio is $\{1,1\}$ which is obviously wrong. However we can get further information about our decision if we calculate the likelihood ratio of the whole codeword corresponding to the information bit. In our example the value will be close to zero which usually means a erroneous decision.

To counter this problem we will make two adjustments. In our algorithm we are trying to optimize the whole problem by looking only at the partial problem. The basis of our algorithm is that the codeword with largest likelihood ratio will also have the largest likelihood ratio value corresponding to the information bits. This may not be entirely true. However it is quite likely that the optimal codeword will have "large" likelihood ratio value corresponding to the information bits. So if we consider all the codewords which have "large" likelihood ratio value then we have a better chance of considering the optimal codeword in our final calculation. The final decision will be obtained after we calculate the likelihood of the whole codeword corresponding to each of the likely information bit sequences. In our example, if we consider the second most likely information bit sequence it is $\{1,0\}$ which corresponds to the correct decision. Thus instead of only the most likely information sequence we will consider several information bits which have large partial likelihood ratio and optimize based on this feasible set.

So far we have been claiming that the information bits are unconstrained. If we look carefully in the codeword space of the $2 / 3$ parity check code, we observe that not only the information bits, but any two out of the three code bits can assume unrestricted value. The third bit is uniquely determined by these two independent bits. We also observe that our confidence in the decision is not constant for all the bits. In our example, we have greater confidence in making decision about the first and the third bits than the second bit. So if we chose our feasible sets based on the received bits for which the likelihood ratio is large, we have a larger probability of including the optimal codeword in our feasible set. The estimated unconstrained 
values corresponding to the first and the third codeword position is $\{1,1\}$, which corresponds to the correct codeword $\{1,0,1\}$.

Generalizing the above idea to a codeword of rate $R$ and block length $N$, we can say that there are $N R$ independent locations. In other words at most $N R$ of $N$ bits can be unconstrained. However which set of $N R$ values can be independent, depends on the type of the code. We want to make our decisions about those $N R$ bits about which we are most confident. In fact, we want to generate $M$ possible partial codewords from which we will obtain the optimal codeword.

Our sub-optimum decoding rule is based on using the sign detector for only $N R$ independent bits of the $N$ coded bits. The remaining $N(1-R)$ bits of $\mathbf{d}$ can be obtained through the code structure of $\mathcal{C}$. The choice of these $N R$ independent bits will be based on the the weight of the location $i \in\{1, \ldots, N\}$ denoted by $w_{i}$ which is equal to $\left|r_{i}\right|$, the absolute value of the received vector at that location. Since large weight corresponds to lower probability of error in the sign detector, the estimates for these $N R$ bits will be reliable. Effectively we want to chose an independent set of bits that has the largest reliability.

\section{Suboptimal decoding of convolutional codes}

Let us now consider the above discussion in terms of a convolutional code $\mathcal{C}$ of block-length $N$, rate $R$ and memory length $\kappa$. The coded bits are given by $\mathbf{d}=\left\{d_{1}, \cdots, d_{N}\right\}$ and the received signal by $\mathbf{r}=\left\{r_{1}, \cdots, r_{N}\right\}$ and we want to find the codeword that minimizes the probability of decoding error. To reduce the complexity of decoding we will make decisions about $N R$ independent bits in an unconstrained manner and then reconstruct the codeword from these $N R$ bits. We would also like to make decisions about those $N R$ bits which can be estimated with largest reliability out of the length $N$ received vector. Simply choosing the largest weight $N R$ locations will not work, as all subsets of $N R$ bits are not independent.

Let $\mathcal{N}=\{1, \cdots, N\}$ represent the set of locations of the coded bits. A set $I \subset \mathcal{N}$ is defined to be an independent subset of $\mathcal{N}$ if the bits corresponding to locations in $I$ can be chosen independently without violating any of the conditions for being part of a codeword in $\mathcal{C}$.

A set $J$ is said to be maximally independent, if it is an independent subset of $\mathcal{N}$ and there does not exist any element $e$, which is in $\mathcal{N}$ but not in $J$ such that $J+e$ is also an independent subset. In our subsequent discussion we will use $I, J, \mathcal{N}$ to represent not only the set of locations but also the coded bits corresponding to those locations when there is no ambiguity. Also as set operators ' + ' will denote the set union and '-' the set difference operation.

In case of a convolutional code of block length $N$ the maximally independent subset of bits not only represent those which can be determined independently, without violating the properties of convolutional code, but also given the values for bits in the maximally independent subset the entire codeword can be uniquely determined. In other words the bits in the maximally independent subset of a convolutional code form a basis for the codeword space. Obviously, for a systematic code word, the set of information bits form a basis for the codewords. In fact a convolutional code is described by a set of relations that define the parity bits in terms of the information bits.

We claim that the basis for a convolutional code is not unique. We will propose an algorithm to calculate the maximum weight basis and we will show it is easy to regenerate the codewords from this basis given the original representation of the convolutional code in terms of the information bits.

For the sake of clarity and simplicity of discussions in the following sections, let us consider a numerical example of a systematic convolutional code of rate $R=1 / 2$, constraint length $\kappa=3$ with the parity bits being defined by the following set of equations,

$$
\left[\begin{array}{c}
b_{2} \\
b_{4} \\
b_{6} \\
b_{8} \\
\vdots \\
b_{N}
\end{array}\right]=\left[\begin{array}{cccccc}
1 & 0 & 0 & 0 & \cdots & 0 \\
1 & 1 & 0 & 0 & \cdots & 0 \\
1 & 1 & 1 & 0 & \cdots & 0 \\
0 & 1 & 1 & 1 & \cdots & 0 \\
\vdots & \ddots & \ddots & \ddots & \ddots & \vdots \\
0 & \cdots & 0 & 1 & 1 & 1
\end{array}\right]\left[\begin{array}{c}
b_{1} \\
b_{3} \\
b_{5} \\
b_{7} \\
\vdots \\
b_{N-1}
\end{array}\right]
$$

where the bits $b_{1}, b_{3}, \cdots, b_{N-1}$ are the information bits and the system is described in $G F(2)$. 
Lemma 1 Any independent subset satisfy the following properties.

1. For a convolutionally coded system of block length $N$ and rate $R$ there are at most $N R$ locations whose values can be chosen independently. In other words the rank of a convolutional code of block-length $N$ and rate $R$ is $N R$.

2. If $I^{\prime} \subset I$, and $I$ is an independent subset of $\mathcal{N}$, then so is $I^{\prime}$.

3. The cardinality of all maximally independent subsets are equal.

Proof: : The second property is true from the definition of the independent subset. We know that convolutional code is a linear code, i.e it is defined by a linear system of equations. The number of independent locations in a convolutional code is given by the rank of the linear system. In general for a systematic convolutional code there are $N(1-R)$ equations involving $N$ variables and the above representation shows that the rank of the system is $N R$. Thus at most $N R$ locations can be chosen independently. In fact the third property says that exactly $N R$ locations can be chosen independently, which is true because any basis of a linear system will have the same number of elements.

We define the weight of a particular location $i, w t(i)$, to be a measure of reliability of making decision on that particular bit location. Quantitatively the non-negative value $\left|r_{i}\right|$ is defined as the weight of that particular bit location. The weight of a set $I$ is given the sum of the weights of each individual member of the set $w t(I)=\sum_{i \in I} w t(i)$.

\subsection{Maximum weight basis}

Our original objective of finding the largest number of independent bits that we can decode most reliably is thus equivalent to finding an independent subset such that given the received vector $\mathbf{r}$ the weight of the subset is maximized. It is clear that since all the weights $w t(i) \geq 0$, our chosen set must be a maximally independent subset; otherwise we could add elements to it without violating the independence condition and thereby form another independent subset whose weight is larger than that of our set. This basis is defined as the maximum weight basis (MWB), $J^{\star}$, of $\mathcal{N}$ with respect to $\mathbf{r}$.

We now present an algorithm to find the MWB $J^{\star}$ of $\mathcal{N}$ with respect to $\mathbf{r}$.

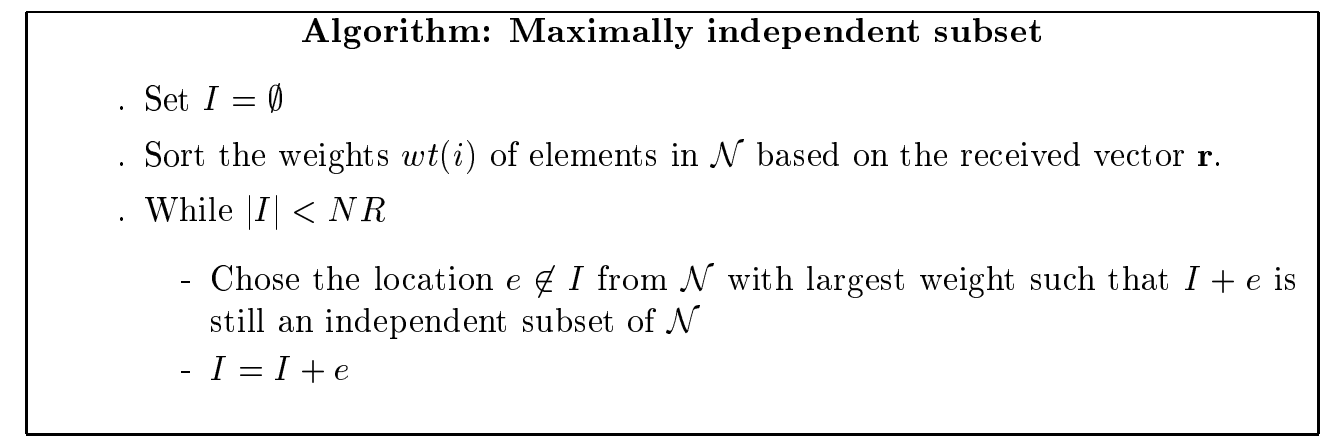

Lemma 2 The above algorithm gives the $M W B$ of $\mathcal{N}$.

Proof: Suppose the above lemma is false. Let $J^{\star}=\left\{e_{1}, e_{2}, \cdots, e_{k}\right\}$ be the maximal subset given by the above algorithm and let $J=\left\{q_{1}, q_{2}, \cdots, q_{k}\right\}$ be a maximally independent subset with total weight larger than $J^{\star}$. Since $J^{\star}$ and $J$ are both maximally independent subsets of $\mathcal{N}$ their cardinality, $k$, are the same.

We assume $w t\left(e_{1}\right) \geq w t\left(e_{2}\right) \geq \cdots \geq w t\left(e_{k}\right)$ and $w t\left(q_{1}\right) \geq w t\left(q_{2}\right) \geq \cdots w t\left(q_{k}\right)$. Of course there might be some elements common in $J^{\star}$ and $J$. Chose the least index $m$ such that $w t\left(q_{m}\right)>w t\left(e_{m}\right)$. Thus the element added at the $m^{t h}$ step is not one of $q_{1}, q_{2}, \cdots, q_{m}$ and for $1 \leq i \leq m$, either $q_{i} \in\left\{e_{1}, \cdots, e_{m-1}\right\}$ or $\left\{e_{1}, \cdots, e_{m-1}, q_{i}\right\}$ is not an independent subset of $\mathcal{N}$.

In other words the set $\left\{e_{1}, \cdots, e_{m-1}\right\}$ of cardinality $(m-1)$ is a maximally independent subset of $\left\{e_{1}, \cdots, e_{m-1}, q_{1}, \cdots q_{m}\right\}$. But the set $\left\{q_{1}, \cdots, q_{m}\right\}$ is an independent subset of $\left\{e_{1}, \cdots, e_{m-1}, q_{1}, \cdots q_{m}\right\}$ 
and is of cardinality $m$. This contradicts the third property that the cardinality of all maximally independent subsets are equal. This proves that our assumption is wrong and our algorithm gives the desired maximally independent subset.

In a convolutional code, deciding whether a particular subset is independent or not and generating codewords from $\mathcal{C}$ based on an independent subset requires some extra observations. A convolutional code can be represented by a system of linear equations relating the coded bits to the information bits. For any equation involving $n$ variables, as soon as $n-1$ of these variables have been determined, the last variable can be found uniquely. Therefore, the locations corresponding to these $n$ variables cannot form an independent subset.

Based on these observations, we next present an algorithm that selects the maximum weight independent subset along with the associated codeword.

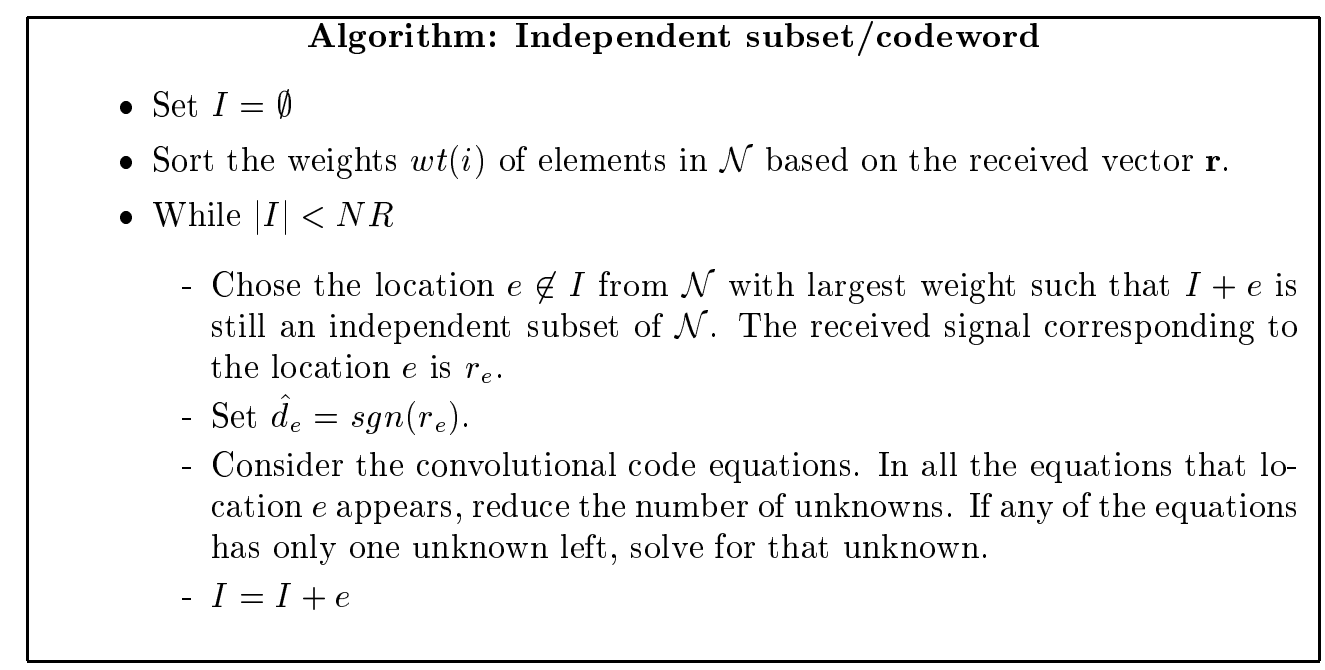

\subsection{M largest weight bases}

At this point we would like to re-emphasize that our final aim is to find an approximate solution to the optimization problem described by (1). Our faith is that the solution obtained by finding the MWB will be close to the optimal solution. However it is intuitive that instead of finding one basis that has a large reliability if we can form a feasible set of a number of large weight bases and then optimize on that particular set, our probability of not finding the optimal codeword will be reduced. In other words, we want to find a set of $M$ bases that have large associated weights and then find the one that gives the highest likelihood among these $M$ bases.

So our first goal is to find the set, $\mathcal{M}_{M}$, of $M$ bases such that there exists no other basis which is not in $\mathcal{M}_{M}$ and whose weight is larger than any basis in $\mathcal{M}_{M}$. We call this set the $M$ largest weight bases. It is evident that $\mathcal{M}_{1}=\left\{J^{\star}\right\}$. We have already found an algorithm to find the MWB $J^{\star}$ and we want to find the other bases from it. We will show that the bases in $\mathcal{M}_{M}$ differ from $J^{\star}$ in a limited number of positions and we can obtain them by modifying the MWB.

Before proceeding any further, let us revisit (2). This relation shows that for any systematic convolutional code we can express all the parity bits in terms of the information bits. If $\mathbf{d}^{P}$ are the parity bits and $\mathbf{d}^{I}$ are the information bits then the original convolutional code relationship can be expressed as $I_{0} \mathbf{d}^{P}=R \mathbf{d}^{I}$ where $I_{0}$ is an identity matrix. Let the bits of the MWB be $\mathbf{d}^{J}$ and the rest of the dependent bits be $\mathbf{d}^{D}$. The original bits $\mathbf{d}^{P}$ and $\mathbf{d}^{I}$ can be each divided into two sets $\mathbf{d}_{1}^{P}, \mathbf{d}_{2}^{P}$ and $\mathbf{d}_{1}^{I}, \mathbf{d}_{2}^{I}$ where the subscript 1 and 2 represent the bits in $\mathbf{d}^{D}$ and $\mathbf{d}^{J}$ respectively. Thus exchanging the columns we can represent the new set of relationships as

$$
\left[\begin{array}{ll}
I_{1} & R_{1}
\end{array}\right] \underbrace{\left[\begin{array}{c}
\mathbf{d}_{1}^{P} \\
\mathbf{d}_{1}^{I}
\end{array}\right]}_{\mathbf{d}^{D}}=\left[\begin{array}{ll}
I_{2} & R_{2}
\end{array}\right] \underbrace{\left[\begin{array}{c}
\mathbf{d}_{2}^{P} \\
\mathbf{d}_{2}^{I}
\end{array}\right]}_{\mathbf{d}^{J}}
$$


We can reduce this relationship as

$$
b_{D}=R^{J} b^{J}
$$

using the algorithm described above. This set of equations uniquely express the dependent bits in terms of the MWB and can also be used to find the values of the parity bits when the values of the bits corresponding to the MWB is obtained.

Lemma 3 For a convolutional code of block-length $N$ and rate $R$ let $J^{\star}$ be the $M W B$. If e and $f$ are two locations in the codeword such that $e \notin J^{\star}, f \in J^{\star}$ and $\left(J^{\star}-f+e\right)$ is also a basis, then wt $(e) \leq w t(f)$.

Proof: $J^{\star}$ is the MWB and $J=\left(J^{\star}-f+e\right)$ is also another basis. This implies

$$
w t\left(J^{\star}\right) \geq w t(J)=w t\left(J^{\star}\right)+w t(e)-w t(f) .
$$

Since all the weights are non-negative $w t(e) \leq w t(f)$

\subsection{Distance of $m^{\text {th }}$ largest basis from maximum weight basis}

Our next effort will be directed towards quantifying the number of places the $m^{\text {th }}$ largest weight basis differs from the MWB. We will give a constructive proof towards our claim. We will first establish a partial ordering among the bases and will show that the MWB act as a least upper bound for each partial ordering.

Lemma 4 If $J_{m}$ is a basis of $\mathcal{N}$, there is a monotone sequence consisting of single exchanges from $J_{m}$ to the $M W B$. In other words if $J_{0}=J^{\star}=\left\{e_{1}, e_{2}, \cdots, e_{k}\right\}$ is the $M W B$ and $J_{m}$ is another basis which differs from $J_{0}$ in $m$ places, then we can find a sequence of bases $J_{1}, J_{2}, \cdots, J_{m-1}$ such that $J_{i}$ differ from $J_{i-1}$ in only one place and $w t\left(J_{m}\right) \leq w t\left(J_{m-1}\right) \leq \cdots \leq w t\left(J_{1}\right) \leq w t\left(J_{0}\right)$.

Proof: From the results of Lemma 1 the number of elements in $J_{i}, i \in\{0,1, \cdots, m\}$ are equal. Let us first consider the bases $J_{0}$ and $J_{m}$. In general there will be some elements which will be common to both the bases and let $S$ be the set of common elements. We will use $S_{0}$ to represent the set of elements $\left\{e_{1}, e_{2}, \cdots e_{m}\right\}$ which are exclusively in $J_{0}$ and not in $J_{m}$ and $S_{m}=\left\{f_{1}, f_{2}, \cdots, f_{m}\right\}$ to represent the elements only in $J_{m}$. We can write

$$
J_{0}=S \cup S_{0} \quad J_{m}=S \cup S_{m} .
$$

$J_{m}$ is a basis and that means any bit in the codeword can be represented uniquely by the elements of $J_{m}$ only. This implies that all the bits in the basis $J_{0}$ can also be represented by the basis $J_{m}$. Now all the elements in $S$ of $J_{0}$ are also elements of $J_{m}$ and can be expressed solely by the corresponding elements in $J_{m}$. Now since the elements of $S_{0}$ are independent of $S$ they cannot be expressed solely by them. In other words to represent the elements $e_{i} \in S_{0}$ we have to use elements from $S_{m}$.

Let $T_{0} \subset S_{0}$ be the elements that use the element $f_{1}$ for its representation. We claim that $T_{0}$ is nonempty. Let us assume that this is not true. This implies that all the elements of $S_{0}$ can be expressed by $S$ and $S_{m}-f_{1}$. Now we have already shown that the elements of $S$ can be solely represented by elements of $S$ only. Combining, all the elements of $J_{0}$ can be represented by $S+S_{m}-f_{1}$. That is $S+S_{m}-f_{1}$ is a basis of $J_{0}$ which is also a basis of $\mathcal{N}$. This implies $S+S_{m}-f_{1}$ is a basis of $\mathcal{N}$ and it has one less element than $J_{m}$. Thus we have two bases of $\mathcal{N}, J_{m}$ and $J_{m}-f_{1}$ with unequal number of elements. This contradicts the third proposition of Lemma 1 and hence our assumption is false. This implies that $T_{0}$ has at least one element. Without loss of generality let us assume that $T_{0}=\left\{e_{1}, \cdots, e_{q}\right\}$.

Let us consider the representation of $e_{i} \in T_{0}$ in terms of the basis set $J_{m}$. This is essentially an equation involving $e_{i}, f_{1}$ and zero or more elements from $J_{m}$. This relationship also suggests how $f_{1}$ can be expressed by the other elements in $J_{m}$ and $e_{i}$. Thus all the elements in $J_{m}$ can be expressed by elements of $J_{m}-f_{1}$ and $e_{i}$. In other words for all $e_{i} \in T_{0}, J_{m}-f_{1}+e_{i}$ forms a basis. All these bases are obtained by a single exchange of element in $J_{m}$ and differ from the MWB, $J_{0}$, at $m-1$ locations. We need to prove that at least one of these bases have weight no less than $J_{m}$ to prove the lemma.

We now try to express $f_{1}$ in terms of the basis elements in $J_{0}$. We claim in this representation there will be at least one element from $T_{0}$. If this is not true, it implies that, $f_{1}$ can be represented by elements from $S+S_{0}-T_{0}$. Now $T_{0}$ is the subset of elements in $J_{0}$ that need $f_{1}$ for their representation in terms of 
the elements of the basis $J_{m}$. That is all the elements in $S+S_{0}-T_{0}$ can be represented solely by $J_{m}-f_{1}$. Thus $f_{1}$ can be represented in terms of elements in $J_{m}-f_{1}$. But $f_{1}$ is a member of the basis $J_{m}$ and as such we should not be able to represent $f_{1}$ by other elements in $J_{m}$. So our assumption is false and in the representation of $f_{1}$ in terms of elements of $J_{0}$, there is at least one element from $T_{0}$. Let that element be $e_{1}^{\prime}$. Using similar as before we can now show that $J_{0}+f_{1}-e_{1}^{\prime}$ is a basis.

That is we have shown that along with $J_{0}$ and $J_{m}$, both $J_{0}+f_{1}-e_{1}^{\prime}$ and $J_{m-1}=J_{0}-f_{1}+e_{1}^{\prime}$ are also bases of $\mathcal{N}$. Now since $J_{0}$ is the MWB and $J_{0}+f_{1}-e_{1}^{\prime}$ is also a basis, from Lemma 3, we can say that weight of $e_{1}^{\prime}$ is no less than weight of $f_{1}$. Thus we have created a new single exchange basis $J_{m-1}=\left(J_{m}-e_{1}^{\prime}+f_{1}\right)$, whose weight is no less than that of $J_{m}$ and which has $(m-1)$ elements different from $J_{0}$. Since $m$ is finite, proceeding in this way, we will be able to generate the monotone sequence.

Lemma 5 If $\mathcal{M}_{m}$ is the set of $m$ maximum weight bases and the basis $J\left(\notin \mathcal{M}_{m}\right)$ is such that the no other basis which is not in $\mathcal{M}_{m}$ has a weight larger than or equal to $J$, then there exists at least one basis $J^{\prime} \in \mathcal{M}_{m}$ such that $J$ differs from $J^{\prime}$ in exactly one location.

Proof: Let us assume that the claim is not true. From the previous lemma it follows that we can find a monotone sequence of single exchanges from $J$ to the MWB $J^{\star}$. Let $J_{1}$ be the basis that is obtained from $J$ by the first single exchange and so $w t\left(J_{1}\right) \geq w t(J)$. Now since $J_{1}$ differs from $J$ in one position it is not an element of $\mathcal{M}_{m}$. This implies there exists a basis $J_{1}$, not in $\mathcal{M}_{m}$, whose weight is greater than or equal to $J$. This contradicts our assumption and asserts the lemma.

From the discussions in the previous section it is evident that for any basis $J_{m}$ which differs from the MWB, $J^{\star}$, at $m$ locations there are at least $m$ bases whose weight are at least as much as $J_{m}$. So the $m^{t h}$ largest basis cannot differ from the MWB at more than $m$ locations. However we will prove a tighter bound on the number of locations in which the $m^{\text {th }}$ largest basis differ from the optimal basis.

Lemma 6 There exist a $m^{\text {th }} M W B$, that differs from the maximum weight basis in at most $\left\lceil\log _{2} m\right\rceil$ locations.

Proof: In order to prove the lemma let us look at a basis $J_{m}$ which differs from the MWB at $m$ locations. Let a chain of single exchange monotone sequence be $J_{M}, J_{m-1}, \cdots, J_{0}=J^{\star}$. As before let us assume that the common elements in $J_{0}$ and $J_{m}$ be $S$ and the differing elements be the sets $S_{0}=\left\{e_{1}, e_{2}, \cdots, e_{m}\right\}$ and $S_{m}=\left\{f_{1}, f_{2}, \cdots, f_{m}\right\}$ respectively.

We have ordered the elements in $S_{0}$ and $S_{m}$ in such a way that $J_{m-i}$ is obtained from $J_{m-i+1}$ by exchanging $f_{m-i}$ with $e_{m-i}$. In particular $J_{1}=J_{0}-e_{1}+f_{1}$ and $J_{2}=J_{1}-e_{2}+f_{2}=J_{0}-e_{1}-e_{2}+f_{1}+f_{2}$ and so on. Following the arguments from the previous lemma, we can show that $w t\left(e_{i}\right) \geq w t\left(f_{i}\right)$ and that $J_{0}-e_{2}+f_{2}$ is also a basis. We can conclude that $w t\left(J_{0}-e_{2}+f_{2}\right) \geq w t\left(J_{2}\right)$. A generalization of this statement is that for every basis $J_{m}$ which differs from the MWB at $m$ locations there are $1+2+\cdots+2^{m-1}=2^{m}-1$ bases which have weight at least as large as $J_{m}$. In other words the $m^{\text {th }}$ largest weight basis differs from the MWB in at most $\left\lceil\log _{2} m\right\rceil$ locations.

\subsection{Replacement elements}

Even though none of the bases in $\mathcal{M}_{M}$ differ from the optimal basis $J^{\star}$ in more than $k=\left\lceil\log _{2} M\right\rceil \operatorname{locations}$ all of them may not necessarily differ from the maximum weight basis at the same $k$ locations. For example it may so happen that all the bases differ from the MWB in one location only but that location is different for each basis. However we can safely claim that the total number of locations in which the optimal basis differ from the other bases in $\mathcal{M}_{M}$ will not exceed $M$. However there are $N R$ possible locations in the codeword. And as such we might need to consider $\left(\begin{array}{c}N R \\ M\end{array}\right)$ locations. Similarly these locations should be filled up with elements from $\mathcal{N}-J \star$. There are $N-N R$ such elements and thus there are $\left(\begin{array}{c}N-N R \\ M\end{array}\right)$ choices. This can be quite large for large codeword sizes. We would like to prune down the options which should be verified to obtain the desired M-largest weight bases.

A pair of elements $[e, f]$ is defined to be a $\mathbf{J}$-exchange such that $e \in J^{\star}$ and $f \in \mathcal{N}-J^{\star}$ and $\left(J^{\star}-e+f\right)$ is a basis. It is apparent that for every element $e \in J^{\star}$ there is a set of elements $R(e)=\{f \mid[e, f]$ is a J-exchange $\}$. We call this set the replacement set of $e$. Of all the members of the replacement set and there is a replacement element, $r(e)=f \in R(e)$, such that among all the elements of $R(e), w t(e)-w t(f)$ is 
the minimum. Just as we have defined the replacement set for the elements in the MWB we can define replacement set, $\tilde{R}(f)$, and replacement element, $\tilde{r}(f)$ for the element $f \in \mathcal{N}-J^{\star}$

Armed with the above informations we are now ready to find the elements of the $M$ maximum weight bases $\mathcal{M}_{M}$. We will first claim that there are a number of locations in the MWB that will remain invariant in all the $M$ bases which are elements of $\mathcal{M}_{M}$. Similarly there will be some elements in $\mathcal{N}-J^{\star}$ that cannot appear in any of the bases. We will also methods to identify them.

Lemma 7 Given a maximum weight basis $J^{\star}$ of $\mathcal{N}$ and the replacement elements $r(e)$ for all elements in $J^{\star}$ the set of $N R-M$ elements that will remain unchanged can be computed in linear time.

Proof: For each element $e \in J^{\star}$ let $w^{\prime}(e)=w t(e)-w t(r(e))$. In other words $w^{\prime}(e)$ is the minimum weight that we lose by replacing the element $e$ from the optimal basis. We can find the $M-1$ smallest values of $w^{\prime}$ using a linear time selection algorithm [5]. Let $S$ be the set of the remaining $N R-M$ elements.

Then for each element $e \in S$ there are at least $M-1$ elements $e^{\prime}$ with $w t\left(J^{\star}-e^{\prime}+r\left(e^{\prime}\right)\right) \leq w t\left(J^{\star}-e+r(e)\right)$, and therefore together with $J^{\star}$ there are at least $M$ bases better than $\left(J^{\star}-e+r(e)\right)$. Therefore every basis in the $M$ maximum weight basis must contain the element $e$.

Similarly for every element $f \in \mathcal{N}-J^{\star}$ we can find the replacement element $R_{\mathcal{N}, J}(f) \in J^{\star}$ and we have a similar lemmas.

Lemma 8 Given a maximum weight basis $J^{\star}$ of $\mathcal{N}$ and the replacement elements $R(f)$ for all elements in $\mathcal{N}-J^{\star}$ the set of $(N-N R-M)$ elements that cannot be a part of any basis of the $M$ maximum weight basis can be calculated.

We have so far shown how to efficiently calculate the MWB $J^{\star}$ of $\mathcal{N}$ and given an optimal weight basis and replacement elements for both the basis and non basis elements how to calculate the set of $M$ elements in $J^{\star}$ that can be potentially replaced and the set of $M$ elements in $\mathcal{N}-J^{\star}$ which are the corresponding potential replacement candidates. However it is yet to be proved how we can calculate the replacement elements and in general the replacement set for every element. We will use two properties to calculate the replacement set for each element both in $J^{\star}$ and $\mathcal{N}-J^{\star}$.

We claim that for all the non basis elements (3) defines the replacement set. Each variable in the non-basis element is uniquely defined in terms of a set of basis variables. We claim that these basis variables constitute the replacement set. If this is not true then let $f_{i}$ be a non basis element which is defined by the basis elements $e_{1}, \cdots, e_{k}$. If $e_{k+1}$ is also a member of the replacement set then it implies that $\left\{e_{1}, \cdots, e_{k}, f_{i}, e_{k+2} \cdots, e_{n}\right\}$ is a basis, where we assumed the rank is $n$. We should be able to describe $e_{k+1}$ in terms of this new basis. Now since $e_{k+1}$ is a part of the MWB it cannot be described exclusively by the basis elements $e_{i}$ and needs $f_{i}$. From this relation we have two descriptions of $f_{i}$ in terms of the optimal basis, one that includes $e_{k+1}$ and the other that doesn't. Thus the description is non-unique which is contradictory to the definition of basis. This proves that the replacement set can be defined by (3).

We also claim that the replacement set relation is symmetric. By that we mean if $f_{i}$ is a member of the replacement set of $e_{j}$ then $e_{j}$ is a member of the replacement set of $f_{i}$. So once we have constructed the replacement set of all the non basis elements we can automatically calculate the replacement set of all basis elements. In terms of (3) the non zero entry in the columns give the replacement set of the basis elements.

It should be noted that given the MWB $J^{\star}$ the two sets together form a necessary and sufficient statistic for the calculation of the $M$ MWB. The set of $M$ elements from $J^{\star}$ and the corresponding replacement elements alone are not enough for the computation as there might be two elements $e$ and $e^{\prime}$ in this set such that there exists two elements $e_{1}, e_{2}$ in the replacement set of $e$ and $w t(e)-w t\left(e_{1}\right)$ and $w t(e)-w t\left(e_{2}\right)$ are both smaller than $w t\left(e^{\prime}\right)-w t\left(r\left(e^{\prime}\right)\right)$ and thus we have to consider both $e_{1}$ and $e_{2}$ before replacing $e^{\prime}$.

Once we have calculated the MWB $J^{\star}$, the $M$ potential replaceable candidates in $J^{\star}$ and $\mathcal{N}-J^{\star}$, we prune the replacement sets to include only these candidates. Thus for each of the $M$ basis elements $e_{i}$ that can be replaced, we calculate $w t\left(e_{i}\right)-w t\left(f_{j}\right)$ where $f_{j}$ is member of both the replacement set of $e_{i}$ and is one of the $M$ elements in the non basis set which are candidates for replacement.

To summarize, in our decoding algorithm, we perform the following steps:

- Chose the maximum weight basis based on the algorithm described in section 3.1 . 
- Calculate the replacement elements of all elements in the basis and the non basis set.

- Select the $M$ elements from both the basis and non-basis set that has the least amount of replacement penalty.

- Calculate the table of replacement penalty for these two set of elements.

- Calculate the $M$ largest weight bases from the maximum weight basis and this table.

The algorithm performs $N \kappa^{2}$ operations to calculate the maximum weight basis and the replacement sets. For each of the $M$ basis, it needs to perform $N$ operations. Thus the total complexity of the decoding algorithm grows only quadratically with the constraint length.

\section{Simulation results}

In this section we present some initial numerical results of our new algorithm. We compare the performance of the suboptimal channel decoding algorithm with Viterbi algorithm for one user. The convolutional code has rate $1 / 2$ and constraint length 3 . We decode 100 information bits at a time. Our simulations have $M=2$, that is we only have kept a codeword list of size 2 in the suboptimal algorithm. Our simulation results show there is very little performance loss in a single user system even when we significantly prune down the computation. However we believe for codes with larger constraint length the size of the set will increase. Such a study is planned as a future work.

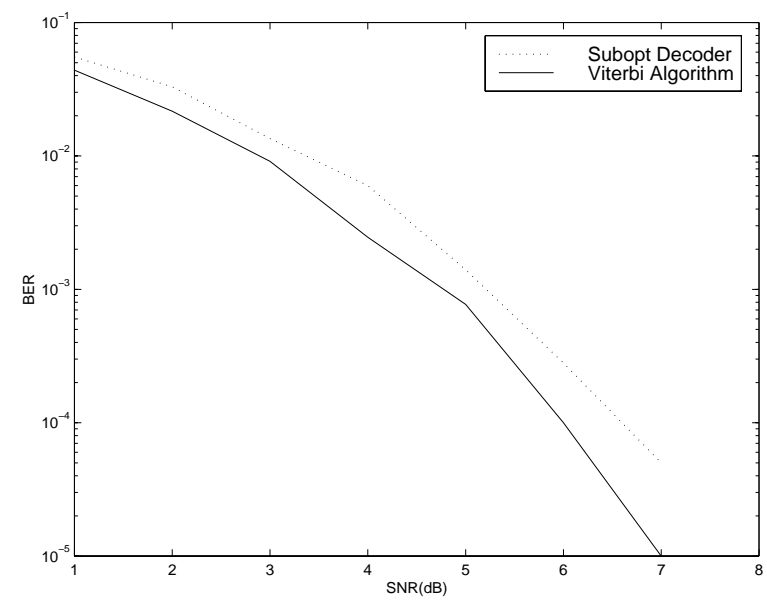

Figure 1: Suboptimal decoding algorithm versus Viterbi decoding

\section{Summary}

The optimal Viterbi decoding algorithm for convolutional codes has a complexity that grows exponentially with the constraint length. This prohibits implementation of "strong" convolutional codes for a practical system. In this paper we have proposed an algorithm that has a complexity quadratic in the constraint length size and yet performs close to the optimal decoding algorithm.

We have only presented the algorithm for a systematic convolutional code. However the results can be easily extended to the non-systematic codes. In this paper we have only investigated situations where we make "hard decisions" on the independent bits. More benefits can probably be gained if we make soft decisions. Finally we would like to extend our results to turbo codes. 


\section{References}

[1] A. J. Viterbi. Error Bound on Convolutional Codes and an Asymptotically Optimmum Decoding Algorithm. IEEE Transactions on Information Theory, 13:260-269, April 1967.

[2] R. M. Fano. A Heuristic Discussion of Probabilistic Decoding. IEEE Transactions on Information Theory, 9:64-74, 1963.

[3] J. L. Massey. Threshold Decoding. MIT Press, Cambridge, MA, 1963.

[4] D. Haccoun and M. J. Ferguson. Generalized Stack Algortihms for Decoding Convolutional Codes. IEEE Transactions on Information Theory, 21:638-651, 1975.

[5] T. H. Cormen, C. E. Leiserson, and R. L. Rivest. Introduction to Algorithms. MIT Press, 1995.

[6] Y. S. Han, C. R. P. hartmann, and C. Chen. Efficient Priority-First Search Maximum-Likelihood SoftDecision Decoding of Linear Block Codes. IEEE Transactions on Information Theory, 39:1514-1523, September 1993.

[7] M. P. C. Fossorier and S. Lin. Soft-Decision Decoding of Linear Block Codes Based on Ordered Statistics. IEEE Transactions on Infromation Theory, 41:1379-1396, September 1995.

[8] K. R. Narayanan and G. L. Stuber. List Decoding of Turbo Codes. IEEE Transactions on Communications, 46(6):754-762, June 1998. 